\title{
OPEN Mycoplasma infection and ocular surface diseases: a nationwide cohort study
}

\author{
Li-Ju Lai ${ }^{1,11}$, Vincent Chin-Hung Chen ${ }^{2,3,11}$, Yao-Hsu Yang ${ }^{4,5,6}$, Kai-Liang Kao7 , Ko-Jung Chen ${ }^{6}$, \\ Ying-Ching Wang ${ }^{8} \&$ Shu-I Wu $u^{9,10 \bowtie ~}$
}

Whether patients with Mycoplasma infection have an increased risk of ocular surface ulcers. Using a nation-wide database, we identified patients with a new diagnosis of Mycoplasma infection between 1997 and 2013, and compared them with age-, sex-, and index year-matched subjects without the infection. Cox proportional regression was performed to compare the risk of corneal diseases between the two cohorts. The incidence of corneal diseases was significantly higher in the 4223 patients with Mycoplasma infection than in the 16,892 patients without ( 7.28 vs. 5.94 per 1000 person-years, $P<0.01$ ). The adjusted hazard ratio for the risk of corneal diseases in the study cohort was 1.21 times higher ( $95 \% \mathrm{Cl} 1.02-1.44)$ than that in the comparison cohort. Mycoplasma infection might be a predisposing factor for patients with keratitis.

Microbial corneal ulcer is a common but potentially sight-threatening ocular infection caused by infectious microorganisms or noninfectious stimuli ${ }^{1}$. Common pathogens include bacterial agents, such as Staphylococcus aureus, Pseudomonas aeruginosa, and Streptococcus pneumonia; fungal agents, such as Fusarium and Aspergillus; and protists. Clinical history taking, detailed clinical examination, or a corneal tissue culture can facilitate pathogen identification ${ }^{2}$. However, the rate of successful laboratory cultivation for microbial corneal ulcer in humans has varied widely from 22 to $75 \%^{3-5}$. The low rates of culture positivity may be due to the lack of corneal scrape samples, the inhibition of microbial growth by antibiotic treatment before corneal tissue culture, the use of inappropriate growth medium, mixed or atypical clinical features, or the coexistence of other pathogens $s^{3,6}$.

Mycoplasma is a recognized pathogen that colonizes the mucosal surfaces of humans and animals ${ }^{7}$. Mycoplasma pneumoniae may cause upper and lower respiratory tract infections in all age groups and is responsible for up to $40 \%$ of community-acquired atypical pneumonia ${ }^{8}$. Extrapulmonary manifestations have been reported in various systems, including the cardiovascular, neurological, dermatological, hematological, musculoskeletal, gastrointestinal, and urogenital systems ${ }^{7,9,10}$. A history of Mycoplasma pulmonary infection or positive findings of serum antibodies are helpful for diagnosing these extrapulmonary infections. Several human case reports of ocular infections caused by Mycoplasma, such as anterior uveitis ${ }^{11-13}$ and papillitis ${ }^{9,14,15}$, have been published. For ocular surface infections, although Mycoplasma is a primary pathogen causing severe infectious keratoconjunctivitis in animals ${ }^{16-18}$, its role as a pathogen in human corneal ulcer has rarely been described. Only few cases of Mycoplasma conjunctivitis have been reported ${ }^{19,20}$. The low rate of detection of Mycoplasma as an etiology for human corneal ulcer can be attributed to the difficulty in obtaining a high rate of culture positivity. Sanchez-Vargas et al. reported that the sensitivity of culture for Mycoplasma in extrapulmonary samples was less than $60 \%{ }^{10}$. Qu et al. reported that only $55.6 \%$ of patients with Mycoplasma pneumoniae showed positive culture results ${ }^{21}$. In addition to direct damage to host cells, previous literature discussed that Mycoplasma-related conjunctivitis or uveitis may be attributed to systemic inflammation or immunological reactions to Mycoplasma ${ }^{7,22,23}$. Studies investigating the association between human ocular surface diseases and Mycoplasma infection are lacking.

\footnotetext{
${ }^{1}$ University Eye Center, Chia-Yi, Taiwan, ROC. ${ }^{2}$ Department of Psychiatry/Health Information and Epidemiology Laboratory, Chang Gung Memorial Hospital, Chia-Yi, Taiwan, ROC. ${ }^{3}$ Department of Psychiatry, Chang Gung University, Tao-Yuan city, Taiwan, ROC. ${ }^{4}$ Department of Traditional Chinese Medicine, Chiayi Chang Gung Memorial Hospital, Chia-Yi, Taiwan, ROC. ${ }^{5}$ School of Traditional Chinese Medicine, College of Medicine, Chang Gung University, Tao-Yuan City, Taiwan, ROC. ${ }^{6} \mathrm{Health}$ Information and Epidemiology Laboratory, Chang Gung Memorial Hospital, Chia-Yi, Taiwan, ROC. 'Department of Pediatrics, Far Eastern Memorial Hospital, New Taipei City, Taiwan, ROC. ${ }^{8}$ Department of Ophthalmology, Taipei City Hospital, Renai Branch, Taipei, Taiwan, ROC. ${ }^{9}$ Department of Medicine, Mackay Medical College, New Taipei City, Taiwan, ROC. ${ }^{10}$ Department of Psychiatry, Mackay Memorial Hospital, Taipei, Taiwan, ROC. ${ }^{11}$ These authors contributed equally: Li-Ju Lai and Vincent Chin-Hung Chen. ${ }^{\circledR}$ email: shuiwu@g.ntu.edu.tw
} 
By using a large, nationwide, population-based database and clinical experience, we conducted a longitudinal cohort study in Taiwan to evaluate whether patients with Mycoplasma infection may have an increased risk of corneal infection.

\section{Methods}

Data source. We used Taiwan's National Health Insurance (NHI) Research Database (NHIRD) to identify and enroll our study and comparison patients. Taiwan launched its single-payer compulsory NHI program in 1995, and this insurance program currently covers $99 \%$ of the population in Taiwan. The NHIRD has been released for research purposes and contains claims data on diagnoses, drug prescriptions, and intervention receipts, but not laboratory results, obtained from ambulatory care, emergency services, and hospitalizations ${ }^{24}$. All personal details on the enrollees of the NHI are encrypted ${ }^{24}$. Diagnoses are coded according to the International Classification of Diseases, Ninth Revision, Clinical Modification (ICD-9-CM) criteria. From the NHIRD, we identified patients who had received a new diagnosis of Mycoplasma pneumonia (M. pneumonia) infection (ICD-9-CM 483.0) (those with first hospitalization and a discharge diagnosis of $M$. pneumoniae infection) between 1997 and 2013. The index date was the date of first hospitalization for M. pneumoniae infection. For each study patient, 4 age-, sex-, index date-, urbanization-, and income-matched comparison patients without $M$. pneumoniae infection were selected. We only included hospitalized patients with a discharge diagnosis of M. pneumoniae infection was because most patients with $M$. pneumoniae infection do not need hospitalization and could be treated in outpatient services. If we include patients from outpatient services, there might be an underestimation for M. pneumoniae infection and an over-estimation for non-infection because not all patients had symptoms or signs serious enough to receive clinical attention. While comparing the hospitalized patients with and without M. pneumoniae infection, we were able to minimize misclassifications of case and comparison groups. Comorbidities were defined as the diagnosis of blepharitis (ICD-9-CM code 373) or glaucoma (ICD9-CM code 365) before the index date. The Charlson Comorbidity Index (CCI) was calculated and compared between the study and comparison patients. Patients with a history of corneal diseases (ICD-9-CM codes 370 and 371) before the index date or those with incomplete age or sex information were excluded.

Main outcome. The main outcome of this study was the date of corneal infection or diseases (ICD-9-CM code 370.xx [keratitis] and 371.xx [corneal opacity and other disorders of the cornea]). All study and comparison patients were followed from the index date until the date of corneal disease diagnoses, withdrawal from the insurance system (i.e. death, jailed, served in the army, or foreigners that exceeded the permitted stay or working permits), loss to follow-up, or the end of the follow-up (31 December 2013), whichever occurred earlier.

Statistical analysis. We used the $\chi^{2}$ test to determine differences in categorical variables and comorbidities before the index date between patients with and without $M$. pneumoniae infection. Student's $t$ test was used to examine the difference in the mean age between the two groups. Incidence rates (per 1000 person-years) for corneal or ocular surface infections stratified by having M. pneumoniae infection, demographic variables, or comorbidities were calculated. The Kaplan-Meier method was used to measure the cumulative incidence, and the log-rank test was used to assess the differences in incidence rates between the 2 groups. Univariable and multivariable Cox proportional hazard regression analyses were performed to estimate and compare crude and adjusted hazard ratios (HRs) and 95\% confidence intervals (CIs) for subsequent corneal diseases between study and comparison patients. All statistical analyses were performed using SAS software (version 9.4, SAS Institute, Cary, NC, USA). The Kaplan-Meier analysis was performed using R software (R Foundation for Statistical Computing, Vienna, Austria). A $P$ value of $<0.05$ was considered statistically significant. All study protocols were approved by the Institutional Review Board of Chang Gung Memorial Hospital (approval no: 201600959B0).

\section{Results}

We included 4223 patients with M. pneumoniae infection in the study cohort and 16,892 age-, sex-, index year-, and sociodemographic-matched patients without $M$. pneumoniae infection in the comparison cohort. Both the study and comparison cohorts showed no significant difference in the distribution of age, sex, urbanization level, or income level (Table 1$)$. The study cohort had significantly higher CCI scores $(P<0.0001)$ and higher blepharitis infection rates $(P=0.001)$ before the index date than did the comparison cohort. The mean latency period between the initial M. pneumoniae infection and the diagnosis of corneal infections or diseases was 5.3 years.

The incidence of corneal infections or diseases was significantly higher in the study cohort than in the comparison cohort (7.28 vs. 5.94, respectively, per 1000 person-years), with an adjusted HR of 1.21 (95\% CI $1.02-1.44, P=0.03$; Table 2). The risk of corneal ulcer or corneal diseases was significantly higher in female and older patients, those who lived in the most urbanized areas, those who had higher CCI scores, and those who had received a diagnosis of blepharitis before the index date.

Figure 1 shows Kaplan-Meier curves for the cumulative incidence of corneal ulcers or diseases between the study and comparison cohorts. A significantly higher risk of corneal diseases was found in the study cohort than in the comparison cohort (log-rank test, $P=0.012$ ).

\section{Discussion}

In the present cohort study, we investigated the association between $M$. pneumoniae infection and subsequent corneal ulcers or diseases. To the best of our knowledge, this is the first population-based longitudinal cohort study to examine such association. After adjusting for sex, age group, income level, urbanization level, CCI score, and comorbidities, the patients with M. pneumoniae infection exhibited a 1.21-fold higher risk of ocular surface diseases, including corneal ulcer, ocular phemphegoid, or cornea degeneration, than those without $M$. 


\begin{tabular}{|c|c|c|c|c|c|}
\hline \multirow[b]{2}{*}{ Variables } & \multicolumn{2}{|c|}{$\begin{array}{l}\text { Mycoplasma } \\
(\mathrm{N}=\mathbf{4 2 2 3})\end{array}$} & \multicolumn{2}{|c|}{$\begin{array}{l}\text { Non- } \\
\text { mycoplasma } \\
(\mathrm{N}=16,892)\end{array}$} & \multirow[b]{2}{*}{ p value } \\
\hline & $\mathbf{n}$ & $\%$ & $\mathbf{n}$ & $\%$ & \\
\hline Gender & & & & & 1.000 \\
\hline Male & 1988 & 47.08 & 7952 & 47.08 & \\
\hline Female & 2235 & 52.92 & 8940 & 52.92 & \\
\hline Age (year) & & & & & 1.000 \\
\hline$<18$ & 2804 & 66.40 & 11,216 & 66.40 & \\
\hline $18-45$ & 779 & 18.45 & 3116 & 18.45 & \\
\hline$>45$ & 640 & 15.16 & 2560 & 15.16 & \\
\hline Urbanization level & & & & & 1.000 \\
\hline 1 (City) & 1112 & 26.33 & 4448 & 26.33 & \\
\hline 2 & 1945 & 46.06 & 7780 & 46.06 & \\
\hline 3 & 801 & 18.97 & 3204 & 18.97 & \\
\hline 4(Villages) & 365 & 8.64 & 1460 & 8.64 & \\
\hline Income & & & & & 1.000 \\
\hline 0 & 3231 & 76.51 & 12,924 & 76.51 & \\
\hline $1 \sim 15,840$ & 255 & 6.04 & 1020 & 6.04 & \\
\hline $15,841 \sim 25,000$ & 439 & 10.40 & 1756 & 10.40 & \\
\hline$>25,000$ & 298 & 7.06 & 1192 & 7.06 & \\
\hline Charlson Comorbidity Index (CCI) & & & & & $<0.001$ \\
\hline$\leqq 2$ & 4055 & 96.02 & 16,597 & 98.25 & \\
\hline$>2$ & 168 & 3.98 & 295 & 1.75 & \\
\hline \multicolumn{6}{|l|}{ Comorbidities } \\
\hline Blepharitis & & & & & 0.001 \\
\hline Yes & 673 & 15.94 & 2352 & 13.92 & \\
\hline No & 3550 & 84.06 & 14,540 & 86.08 & \\
\hline Glaucoma & & & & & 0.055 \\
\hline Yes & 75 & 1.78 & 233 & 1.38 & \\
\hline No & 4148 & 98.22 & 16,659 & 98.62 & \\
\hline
\end{tabular}

Table 1. Demographic characteristics of and comorbidities in patients with and without Mycoplasma pneumonia enrolled in Taiwan's National Health Insurance Research Database.

pneumoniae infection. Such result suggested increased associations between having the history of Mycoplasma infection and subsequent corneal diseases. Mycoplasma may be considered as a precursor for ocular surface predisposition to keratitis, although it is usually neglected because of the difficulty in obtaining culture or DNA confirmations.

Although a strong association between Mycoplasma and infectious keratoconjunctivitis in animals has been described, few human cases of Mycoplasma conjunctivitis have been reported in the literature ${ }^{19,20}$. No previous cohort study has examined the association between human systemic M. pneumoniae infection and subsequent ocular surface involvements. Although the pathomechanisms through which M. pneumoniae causes a wide range of extrapulmonary infections remain largely unknown, the following 3 nonmutually exclusive mechanisms are proposed $^{22}$. (1) Direct Mycoplasma invasion: Since the cornea has no blood vessels, the pathogen of M. pneumoniae may be transmitted through tears or aqueous fluid diffusions to infiltrate the cornea. Such process takes longer time than the hematogenous transmissions. In addition, Mycoplasmal antigens, such as glycoproteins and glycolipids, may activate local inflammatory cytokines (eg, interleukin [IL]-6 and IL-8) to induce inflammatory responses. (2) Indirect immune modulation: the absence of Mycoplasma infection at the site of inflammation, whereas the manifestation in distant organs may be caused through autoimmunity or immune complex formation $^{22}$. (3) Vascular occlusion type: extrapulmonary or neurological manifestations of Mycoplasma infection may be caused by the obstruction of blood flow associated with the formation of thrombosis, embolism, vasculitis, or vasculopathy involving an immune complex (eg, antiphospholipid antibodies $)^{7,22,23}$. However, it remained unclear whether ocular surface involvements in our study patients were due to the direct spread of $M$. pneumoniae to the eyes or a Mycoplasma-induced immune response cross-reacting with the ocular surface tissue. Our finding that the mean latency period between the initial Mycoplasma infection and subsequent corneal disease diagnosis was not immediate but an average of 5 years later suggests possible chronic influences of vascular occlusions involving immune complexes. These observations might be consistent with the aforementioned mechanisms, resulting in complicated clinical manifestations.

The prevalence of microbiology varied in different geographical regions. Research showed that in China, prevalence of viral (43.6\%) or bacterial (46.2\%) keratitis accounted for nearly $90 \%$ of the corneal infection ${ }^{25}$. Whereas in Uganda, over half of the keratitis were fungal (62\%), 7\% were mixed bacterial and fungal, $7 \%$ 


\begin{tabular}{|c|c|c|c|c|c|c|c|c|}
\hline \multirow{3}{*}{$\begin{array}{l}\text { Variables } \\
\text { Mycoplasma (yes/no) }\end{array}$} & \multicolumn{4}{|c|}{ Crude } & \multicolumn{4}{|c|}{ Adjusted $^{\mathrm{a}}$} \\
\hline & \multirow{2}{*}{\begin{tabular}{|l|} 
HR \\
1.23 \\
\end{tabular}} & \multicolumn{2}{|l|}{$95 \%$ CI } & \multirow{2}{*}{\begin{tabular}{|l|} 
p value \\
0.023 \\
\end{tabular}} & \multirow{2}{*}{$\frac{\mathrm{HR}}{1.21}$} & \multicolumn{2}{|c|}{ 95\% CI } & \multirow{2}{*}{\begin{tabular}{|l|} 
p value \\
0.033
\end{tabular}} \\
\hline & & 1.03 & 1.46 & & & 1.02 & 1.44 & \\
\hline Gender (male/female) & 0.78 & 0.67 & 0.91 & 0.001 & 0.80 & 0.68 & 0.93 & 0.003 \\
\hline \multicolumn{9}{|l|}{ Age (year) } \\
\hline$<18$ & 1.00 & \multicolumn{3}{|c|}{ Reference } & 1.00 & \multicolumn{2}{|c|}{ Reference } & \\
\hline $18-45$ & 1.00 & 0.80 & 1.26 & 0.980 & 1.02 & 0.77 & 1.36 & 0.872 \\
\hline$>45$ & 1.70 & 1.38 & 2.09 & $<0.001$ & 1.69 & 1.26 & 2.26 & $<0.001$ \\
\hline \multicolumn{9}{|l|}{ Urbanization level } \\
\hline 1 (City) & 1.72 & 1.25 & 2.35 & 0.001 & 1.68 & 1.22 & 2.31 & 0.001 \\
\hline 2 & 1.25 & 0.91 & 1.70 & 0.167 & 1.24 & 0.91 & 1.69 & 0.184 \\
\hline 3 & 1.12 & 0.79 & 1.57 & 0.535 & 1.09 & 0.77 & 1.53 & 0.633 \\
\hline 4(Villages) & 1.00 & \multicolumn{2}{|c|}{ Reference } & & 1.00 & \multicolumn{2}{|c|}{ Reference } & \\
\hline \multicolumn{9}{|l|}{ Income } \\
\hline 0 & 1.00 & \multicolumn{2}{|c|}{ Reference } & & 1.00 & \multicolumn{2}{|c|}{ Reference } & \\
\hline $1 \sim 15,840$ & 0.93 & 0.65 & 1.35 & 0.710 & 0.79 & 0.53 & 1.18 & 0.246 \\
\hline $15,841 \sim 25,000$ & 1.25 & 0.97 & 1.61 & 0.087 & 0.96 & 0.69 & 1.33 & 0.794 \\
\hline$>25,000$ & 1.34 & 0.99 & 1.82 & 0.056 & 1.05 & 0.72 & 1.52 & 0.806 \\
\hline \multicolumn{9}{|c|}{ Charlson Comorbidity Index (CCI) } \\
\hline$\leqq 2$ & 1.00 & \multicolumn{2}{|c|}{ Reference } & & 1.00 & \multicolumn{2}{|c|}{ Reference } & \\
\hline$>2$ & 2.14 & 1.36 & 3.38 & 0.001 & 1.49 & 0.91 & 2.42 & 0.112 \\
\hline \multicolumn{9}{|l|}{ Comorbidity (yes/no) } \\
\hline Blepharitis & 1.57 & 1.31 & 1.88 & $<0.001$ & 1.56 & 1.30 & 1.87 & $<0.001$ \\
\hline Glaucoma & 2.01 & 1.24 & 3.26 & 0.004 & 1.47 & 0.90 & 2.41 & 0.124 \\
\hline
\end{tabular}

Table 2. Cox model with the hazard ratio (HR) and 95\% confidence interval (CI) of corneal and ocular surface infection with potential covariates. Crude HR: relative hazard ratio; Adjusted HR: adjusted hazard ration, mutually adjusted for age, sex, levels of income and urbanizations, Charlson Comorbidity Index scores, and comorbidities of blepharitis and glaucoma.

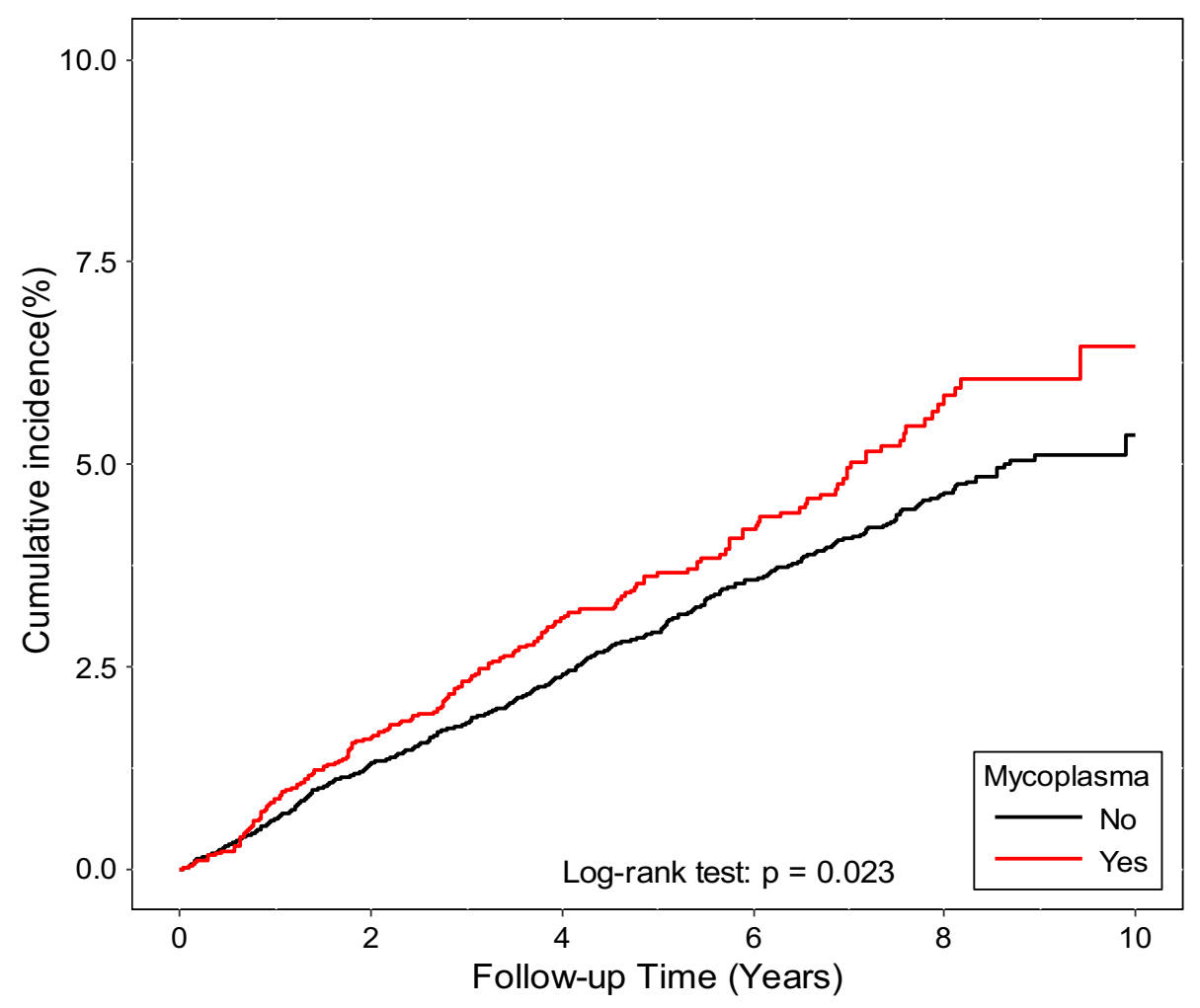

Figure 1. Cumulative incidence of corneal infection in patients with and without Mycoplasma pneumonia infection. 
bacterial, and $24 \%$ no organism detected ${ }^{26}$. Often times, the pathogen identification for corneal ulcer requires history taking, detailed clinical examinations, or the facilitation of corneal tissue culture ${ }^{2}$. However, the rate of successful laboratory cultivation for microbial corneal ulcer in humans has varied widely from 22 to $75 \%{ }^{3-5}$. In bacterial infections, rapid inflammation, dense stromal suppuration, and ground glass appearance on cornea are usually observed. In fungal keratitis, stromal infiltrates with filament edges, satellite lesions, and thick endothelial exudate are often described. The ring-shaped infiltration could also be seen in fungal, herpes simplex, or amoeba keratitis ${ }^{30}$. Mycoplasma infection presented quite differently from typical infections caused by the pathogens mentioned above. Macrolides (eg. azithromycin and clarithromycin) are the treatment of choice for Mycoplasma infection. It can not only eradicate the microorganism but also reduce the amount of potentially harmful bacterial components, such as the bacterial cell wall ${ }^{7}$. Furthermore, macrolides may be beneficial in managing the indirect-type extrapulmonary manifestations of Mycoplasma infection by suppressing the immune responses of the host ${ }^{7,27,28}$. In addition to antibacterial activity, macrolides have immunomodulatory properties than can enhance host defense mechanisms in the first few weeks of drug initiation. Prolonged curtailment of local inflammation or neutrophil activities also helps resolving infection or inflammation ${ }^{29}$. Mycoplasma is may be an overlooked predisposing factor of keratitis, even when the examining tools were not good enough, the ophthalmologist's high degree of clinical suspicion on different manifestations of co-infections and relevant treatments may help give patients a better treatment result.

The strengths of this study include the use of a nationwide database to conduct large longitudinal cohorts with adequate statistical power to detect the association of interest. There are some limitations in this study. First, the results of laboratory tests, culture findings, and imaging studies were not available in the NHIRD; and significant differences were found on underlying comorbidities and blepharitis between the two cohorts. It is also possible that the higher rates of ocular surface disease might be correlated with the underlying comorbidities. Although we have tried to adjust these differences and our result still revealed a significantly higher risk of corneal ulcer in the Mycoplasma infection group within the stratification of people with blepharitis (adjusted HR 1.57, 95\% CI 1.30 1.87, Table 2), we still lack sufficient information to explore possible causal mechanisms in this longitudinal observational study. Future clinical studies with sufficient laboratory assessments are still required. Second, patients with mild Mycoplasma infection might not have been hospitalized for treatment. In accordance with our inclusion criteria, we identified study patients who had Mycoplasma infection sufficiently severe to require hospitalization for clinical management. Hence, our findings might only be generalized to the relatively severely affected subgroups of people. Third, information for the longitudinal cohort was drawn from datasets developed for administrative rather than for research purposes. Diagnoses obtained from these datasets reflect real-world clinical practice and might not necessarily generalize for researching diagnostic criteria. Underor over- estimations for diagnoses of Mycoplasma infection and corneal diseases may occur. However, previous literature has described moderate to substantial agreements between the NHIRD claim data and medical charts (Kappa values of $0.55 \sim 0.86$ ). In addition, all insurance claims (including diagnoses and managements given) were scrutinized regular by peer supervision. If fraud is identified, penalties would be given to physicians. Hence, the diagnoses in our study may be judged as reliable. Fourth, no information on lifetime Mycoplasma infection before the launch of the NHI system could be obtained, and we could conduct analyses using only information obtained during our follow-up window (i.e. 1997 to 2013). Such measurement errors occurred in both exposure and outcome; therefore, the association of interest might have been attenuated rather than exaggerated.

\section{Conclusion}

The results of our population-based observational study revealed an increased association between Mycoplasma infection and subsequent corneal diseases. Mycoplasma infection may be considered as a precursor for ocular surface predisposition to keratitis. Appropriate laboratory examinations, as well as adequate therapeutic managements, may be applied if the possibility of Mycoplasma infection is suspected. Additional large prospective noninterventional studies are required to elucidate the microbiological, immunological, and hematological causal mechanisms ${ }^{22}$.

Received: 7 December 2020; Accepted: 2 November 2021

Published online: 22 November 2021

\section{References}

1. Lakhundi, S., Siddiqui, R. \& Khan, N. A. Pathogenesis of microbial keratitis. Microb. Pathog. 104, 97-109 (2017).

2. Wilhelmus, H. K., Liesegang, T. G., Osato, M. S. \& Jones, D. B. Cumitech 13A: Laboratory Diagnosis of Ocular Infections 157-168 (American Society of Microbiology, 1994).

3. Chidambaram, J. D. et al. Prospective study of the diagnostic accuracy of the in vivo laser scanning confocal microscope for severe microbial keratitis. Ophthalmology 123, 2285-2293 (2016).

4. Ranjini, C. Y. \& Waddepally, V. V. Microbial profile of corneal ulcers in a tertiary care hospital in south India. J. Ophthalmic Vis. Res. 11, 363-367 (2016).

5. Suwal, S., Bhandari, D., Thapa, P., Shrestha, M. K. \& Amatya, J. Microbiological profile of corneal ulcer cases diagnosed in a tertiary care ophthalmological institute in Nepal. BMC Ophthalmol. 16, 209 (2016).

6. Burton, M. J. et al. Microbial keratitis in East Africa: Why are the outcomes so poor?. Ophthalmic Epidemiol. 18, 158-163 (2011).

7. Narita, M. Pathogenesis of extrapulmonary manifestations of Mycoplasma pneumoniae infection with special reference to pneumonia. J. Infect. Chemother. 16, 162-169 (2010).

8. Waites, K. B., Balish, M. F. \& Atkinson, T. P. New insights into the pathogenesis and detection of Mycoplasma pneumoniae infections. Future Microbiol. 3, 635-648 (2008).

9. Yimenicioglu, S., Yakut, A., Ekici, A., Bora Carman, K. \& Cagri Dinleyici, E. Mycoplasma pneumoniae infection with neurologic complications. Iran. J. Pediatr. 24, 647-651 (2014).

10. Sanchez-Vargas, F. M. \& Gomez-Duarte, O. G. Mycoplasma pneumoniae-an emerging extra-pulmonary pathogen. Clin. Microbiol. Infect. 14, 105-117 (2008). 
11. Perry, J. T. \& Chen, W. S. Acute Mycoplasma pneumoniae infection presenting with unilateral anterior uveitis and perineuritis. J. AAPOS. 20(2), 178-180 (2016).

12. Weinstein, O., Shneck, M., Levy, J. \& Lifshitz, T. Bilateral acute anterior uveitis as a presenting symptom of Mycoplasma pneumoniae infection. Can. J. Ophthalmol. 41, 594-595 (2006).

13. Yashar, S. S., Yashar, B., Epstein, E. \& Viani, R. M. Uveitis associated with Mycoplasma pneumoniae meningitis. Acta Ophthalmol. Scand. 79, 100-101 (2001).

14. Chiang, W. Y. \& Huang, H. M. Bilateral monosymptomatic optic neuritis following Mycoplasma pneumoniae infection: A case report and literature review. Indian J. Ophthalmol. 62, 724-727 (2014).

15. Choi, S. Y., Choi, Y. J., Choi, J. H. \& Choi, K. D. Isolated optic neuritis associated with Mycoplasma pneumoniae infection: Report of two cases and literature review. Neurol. Sci. https://doi.org/10.1007/s10072-017-2922-9 (2017).

16. Akerstedt, J. \& Hofshagen, M. Bacteriological investigation of infectious keratoconjunctivitis in Norwegian sheep. Acta Vet. Scand. 45, 19-26 (2004).

17. Angelos, J. A. Infectious bovine keratoconjunctivitis (pinkeye). Vet. Clin. North Am. Food Anim. Pract. 31(61-79), v-vi (2015).

18. Arnal, M. et al. Dynamics of an infectious keratoconjunctivitis outbreak by Mycoplasma conjunctivae on Pyrenean Chamois Rupicapra p. pyrenaica. PLoS ONE 8, e61887 (2013).

19. Bjornelius, E., Jensen, J. S. \& Lidbrink, P. Conjunctivitis associated with Mycoplasma genitalium infection. Clin. Infect. Dis. 39, e67-69 (2004).

20. Jones, D. M. \& Tobin, B. Neonatal eye infections due to Mycoplasma hominis. Br. Med. J. 3, 467-468 (1968).

21. Qu, J. et al. Accuracy of IgM antibody testing, FQ-PCR and culture in laboratory diagnosis of acute infection by Mycoplasma pneumoniae in adults and adolescents with community-acquired pneumonia. BMC Infect. Dis. 13, 172. https://doi.org/10.1186/ 1471-2334-13-172 (2013).

22. Narita, M. Classification of extrapulmonary manifestations due to mycoplasma pneumoniae infection on the basis of possible pathogenesis. Front. Microbiol. 7, 23. https://doi.org/10.3389/fmicb.2016.00023 (2016).

23. Narita, M. Pathogenesis of neurologic manifestations of Mycoplasma pneumoniae infection. Pediatr. Neurol. 41, 159-166 (2009).

24. NHIRD. National Health Insurance Research Database, Taiwan. Introduction to the National Health Insurance Research Database (NHIRD), Taiwan. http://www.nhri.org.tw/nhird/en/index.htm. Accessed 20 January 2010 (2006).

25. Cao, J. et al. Prevalence of infectious keratitis in Central China. BMC Ophthalmol. 14, 43. https://doi.org/10.1186/1471-2415-14-43 (2014).

26. Arunga, S. et al. Epidemiology of microbial keratitis in uganda: A cohort study. Ophthalmic Epidemiol. 27, 121-131. https://doi. org/10.1080/09286586.2019.1700533 (2020).

27. Amsden, G. W. Anti-inflammatory effects of macrolides: An underappreciated benefit in the treatment of community-acquired respiratory tract infections and chronic inflammatory pulmonary conditions?. J. Antimicrob. Chemother. 55, 10-21 (2005).

28. Tagliabue, C. et al. The impact of steroids given with macrolide therapy on experimental Mycoplasma pneumoniae respiratory infection. J. Infect. Dis. 198, 1180-1188 (2008).

29. Culic, O. et al. Azithromycin modulates neutrophil function and circulating inflammatory mediators in healthy human subjects. Eur. J. Pharmacol. 450, 277-289 (2002).

30. Garg, P. \& Rao, G. N. Corneal ulcer: Diagnosis and management. Community Eye Health 12, 21-23 (1999).

\section{Acknowledgements}

The authors thank the Health Information and Epidemiology Laboratory (CLRPG6G0041) for their comments and assistance in data analysis. This study is based in part on data from the National Health Insurance Research Database provided by the Bureau of National Health Insurance, Department of Health, and managed by the Taiwan National Health Research Institutes, Republic of China. SIW is partly funded by Mackay Memorial Hospital (MMH-109-105, MMH-109-108, MMH-109-112).

\section{Author contributions}

All authors have contributed significantly, and all authors are in agreement with the content of the manuscript: Conception/design: V.C.-H.C. and L.-J.L.; Collection and/or assembly of data: All authors; Data analysis and interpretation: All authors; Manuscript writing: S.-I.W., L.-J.L., V.C.-H.C., and K.-L.K.; Final approval of manuscript: All authors.

\section{Funding}

Medical Research Council, Chang-Gung Memorial Hospital.

\section{Competing interests}

The authors declare no competing interests.

\section{Additional information}

Correspondence and requests for materials should be addressed to S.-I.W.

Reprints and permissions information is available at www.nature.com/reprints.

Publisher's note Springer Nature remains neutral with regard to jurisdictional claims in published maps and institutional affiliations.

\footnotetext{
(c) (i) Open Access This article is licensed under a Creative Commons Attribution 4.0 International License, which permits use, sharing, adaptation, distribution and reproduction in any medium or format, as long as you give appropriate credit to the original author(s) and the source, provide a link to the Creative Commons licence, and indicate if changes were made. The images or other third party material in this article are included in the article's Creative Commons licence, unless indicated otherwise in a credit line to the material. If material is not included in the article's Creative Commons licence and your intended use is not permitted by statutory regulation or exceeds the permitted use, you will need to obtain permission directly from the copyright holder. To view a copy of this licence, visit http://creativecommons.org/licenses/by/4.0/.
}

(C) The Author(s) 2021 